\title{
Introduction for the special issue: Fracture healing and bone regeneration
}

\author{
Alesha B. Castillo ${ }^{1,2}$
}

Published online: 26 November 2015

(c) Springer Science+Business Media New York 2015

Successful clinical management of fracture repair requires an understanding of repair biology, advantages and limitations of current fixation methods, and feasibility of and selection criteria for grafting approaches including the use of viable cells, biologics, and physical stimulation. In this issue, Leucht and colleagues provide an overview of fracture repair from a clinical perspective with emphasis on the underlying biology, evaluation and management of segmental defects and non-unions, and up-to-date methods for enhancing repair including physical stimulation, grafts, and biologics. Successful translation of research findings to the clinic requires selection of appropriate experimental models and modalities, and there is a push to utilize large animals in orthopedic research. To this end, Kacena and colleagues discuss current experimental methods, types of fixation, and large animal models used to address long bone segmental defect repair. A second article from the same group discusses strategies for enhancing repair of segmental defects through improved vascularization of grafts and artificial scaffolds using biological, chemical, and tissue-engineered approaches. Fisher and colleagues discuss next-generation scaffolds developed using additive manufacturing technologies, namely 3D bioprinted scaffolds in combination with viable cells, and address their mechanical properties and support of extracellular matrix production and mineralization. Lastly, fracture healing and bone regeneration are acutely sensitive to the prevailing mechanical environment, and Morgan and Lei review the effects of mechanical stimuli on bone repair at the organ, tissue, cellular, and molecular levels. This final paper highlights the importance of understanding the inherent mechanical environment at the injury site, and how regulation of this environment through clinical decision-making (e.g., fixation selection, mechanical properties of grafting materials, and post-operative weight-bearing) can enhance repair of the most challenging cases.

Alesha B. Castillo

alesha.castillo@nyu.edu; alesha.castillo@nyumc.org;

http://castillolabs.com

1 Department of Mechanical Engineering, NYU Tandon School of Engineering, New York University, New York, NY, USA

2 Department of Orthopaedic Surgery, NYU School of Medicine, New York University, New York, NY, USA 